\title{
GIẢI QUYẾT MỘT SỐ VẤN ĐỀ KHOA HỌC - KỸ THUẬT LIÊN QUAN ĐẾN VIỆC XỦ̉ LÝ TOÁN HỌC MẠNG LƯỚI ĐỘ CAO NHÀ NƯỚC TRONG HỆ ĐỘ CAO DỰA TRÊN MẶT GEOID HÒN DẤU
}

\author{
PGS. TSKH. HÀ MINH HÒA, ThS. NGUYẼN TH! THANH HƯƠNG \\ Viện Khoa học Đo đạc và Bản đồ
}

\section{Tóm tắt:}

Bài báo khoa học này định hướng đến việc xây dựng công thức chuyển chênh cao đo từ trọng trường thực của Quả đất thành hiệu các đại lượng địa thế năng giữa hai mốc độ cao phục vụ việc bình sai mạng lưới độ cao quốc gia trong hệ độ cao dựa trên mặt Geoid. Bài báo này cũng chỉ ra rằng khi giá trị trung bình của gia tốc lực trọng trường chuẩn tương ứng với mốc độ cao được biểu diễn trong đơn vị $\mathrm{kGal}$, các hiệu các đại lượng địa thế năng và thế năng trọng trường thực sẽ có đơn vị $k G a l . m$. Trong trường hợp này, các hạn sai được quy định trong Quy phạm lưới độ cao quốc gia sẽ không thay đổi giá trị, chỉ có đơn vị được thay đổi từ mm thành $\mathrm{kGal} . \mathrm{mm}$, từ $\mathrm{mm} / 1 \mathrm{~km}$ thành $\mathrm{kGal} . \mathrm{mm} / 1 \mathrm{~km}$. Tương tự, trọng số của hiệu các đại lượng địa thế năng của đường độ cao bằng trọng số của chênh cao đo của đường độ cao đó.

\section{1. Đặt vấn đề}

$\mathrm{H}$ ệ độ cao dựa trên mặt Geoid đã trở thành hiện thực trong thực tiễn trắc địa quốc tế nhằm thay thế hệ độ cao dựa trên mặt biển trung bình tại trạm nghiệm triều 0 (ở Việt Nam là trạm nghiệm triều Hòn Dâu). Các hệ độ cao dựa trên mặt Geoid của các nước châu Âu EVRF2007 (Sacher M., Ihde J., Liebsch G., M ä kinen J. (2008)) đã hoàn thành năm 2007, của các nước Nam Mỹ SIRGAS2000 (Sánchez, L. (2005); Fortes P., Lauría E., Brunini C., Amaya W., Sánchez L., Drewes H., Seemuller W. (2006)) hoàn thành năm 2000. Phân ban Đo đạc trắc địa (The Geodetic Survey Division - GSD) trực thuộc Bộ Tài nguyên của Canada (Natural Resources Canada - NRCan) đang triển khai Dự án hiện đại hóa hệ độ cao Canada GVRF (Geoid - based Vertical Reference Frame for North America) được bắt đầu từ năm 2002 và dự kiến sẽ kết thúc vào năm 2013 (M. Veronneau, J. Huang. (2007); Sideris Michael G., Spiros Pagiatakis. (2010)). Nước Mỹ đang trù tính phối hợp với Canada xây dựng hệ độ cao dựa trên mặt Geoid và kết thúc vào năm 2022 (Daniel Roman and Neil Weston (2012)). Tiền đề để thúc đẩy việc xây dựng hệ độ cao dựa trên mặt Geoid ở các nước và các khu vực trên thế giới xuất phát từ việc xác định được thế năng trọng trường thực $\bar{W}_{0}=62636856.0 \mathrm{~m}^{2} \cdot \mathrm{s}^{-2}$ của Geoid toàn cầu nhờ phương pháp đo cao từ vệ tinh (altimetry) từ các dự án vệ tinh TOPEX/POSEIDON, JASON1, JASON2, ENVISAT, GFO, GEOSAT... của Mỹ, ERS1, ERS2 ... của châu Âu (Bursa M., Kenyon S., Kouba J., Muller A., Radej K., Vatrt V., Vojtiskova M., Vitek V. (1999); Bursa M., Kenyon S., Kouba J., Radej K., Vatrt V., Vojtiskova M., Simek J. (2002); Bursa M., Kenyon S., Kouba

Người phản biện: TS. Nguyễn Đình Thành 
J., Sima Z., Vatrt V., Vitek V. and Vojtiskova M. (2007)) và được Tổ chức Dịch vụ quay Quả đất IERS (Internatioal Earth Rotation Service) thừa nhận trong các Quyết định 2003 và 2010 (Dennis D. McCarthy, Gerard Petit. (2004); Petit G., Luzum B. (2010) ). Ngoài ra, mặt Geoid toàn cầu này được sử dụng trong nhiều mô hình trọng trường Quả đất GOCE, GRACE, EGM2008, USGG2012 .v.V..., các mô hình Bề mặt động lực trung bình MDT (Mean Dynamic Topography) và các mô hình Mặt biển trung bình MSS (Mean Sea Surface). Các lợi ích của hệ độ cao dựa trên mặt Geoid so với hệ độ cao dựa trên mặt biển trung bình đã được phân tích trong các tài liệu (Hà Minh Hòa (2012a); Hà Minh Hòa (2012b)). Ở Việt Nam, dựa trên phương pháp xác định thế năng trọng trường thực $\mathrm{W}_{0}$ của mặt Geoid cục bộ sát nhất với mặt biển trung bình Hòn Dấu trên cơ sở sử dụng các giá trị độ cao chuẩn trên các điểm độ cao hạng I kết hợp với các kết quả đo đạc, xử lý các dữ liệu GPS và xác định dị thường độ cao từ mô hình trọng trường Quả đất trên các mốc độ cao này được đề xuất trong các tài liệu (Hà Minh Hòa (2007); Hà Minh Hòa (2012c)) đã xác định được thế năng trọng trường thực $\mathrm{W}_{0}=62636847.2911 \mathrm{~m}^{2} \cdot \mathrm{s}^{-2}$ của mặt Geoid cục bộ sát nhất với mặt biển trung bình Hòn Dấu với sai số trung phương $\pm 0.183 \mathrm{~m}^{2} \cdot \mathrm{s}^{-2}$ (xem Hà Minh Hòa (2012d); Hà Minh Hòa và nnk (2012e)). Đây là cơ sở khoa học để định hướng cho việc phát triển hệ độ cao dựa trên mặt Geoid ở Việt Nam.

Việc bình sai mạng lưới độ cao hạng I, II trong hệ độ cao dựa trên mặt Geoid với các tham số ẩn là các đại lượng địa thế năng của các mốc độ cao thay cho các độ cao chuẩn là giải pháp kỹ thuật phù hợp. Trước kia, khi sử dụng mặt biển trung bình tại trạm nghiệm triều 0 làm mặt khởi tính cho hệ độ cao, người ta coi độ cao chuẩn của điểm nằm trên mặt biển trung bình bằng 0 . Từ đây, qua mạng lưới độ cao gốc truyền độ cao từ điểm với độ cao chuẩn bằng 0 trên mặt biển trung bình đến điểm khởi tính của mạng lưới độ cao hạng I, II quốc gia. Khi sử dụng mặt Geoid sát nhất với mặt biển trung bình tại trạm nghiệm triều 0 làm mặt khởi tính cho hệ độ cao dựa trên mặt Geoid, lưu ý thế năng trọng trường thực của mặt Geoid này bằng $W_{0}$, chúng ta xác định đại lượng địa thế năng $\mathrm{C}_{\mathrm{M}}$ của mốc độ cao $M$ theo công thức $C_{M}=W_{0}-W_{M}$, ở đây $W_{M}$ là thế năng trọng trường thực của mốc độ cao $\mathrm{M}$. Khi thay cho độ cao chuẩn $H_{M}^{\gamma}$ của mốc độ cao $\mathrm{M}$ bằng đại lượng địa thế năng $\mathrm{C}_{\mathrm{M}}$ của nó, chúng ta lưu ý mối quan hệ giữa chúng:

$$
H_{M}^{\gamma}=\frac{C_{M}}{\bar{\gamma}_{M}},
$$

ở đây $\bar{\gamma}_{M}$ - giá trị trung bình của gia tốc lực trọng trường chuẩn tương ứng với mốc độ cao M.

Việc xác định đại lượng địa thế năng $\mathrm{C}_{\mathrm{M}}$ thay cho độ cao chuẩn $H_{M}^{\gamma}$ của mốc độ cao $\mathrm{M}$ có lợi ích to lớn không chỉ đối với việc giải quyết bài toán chuyển đổi độ cao chuẩn giữa các hệ độ cao dựa trên mặt Geoid, mà còn cung cấp dữ liệu bổ sung quan trọng để làm chính xác các hệ số khai triển điều hòa của thế năng trọng trường Quả đất, ngoài dữ liệu đo trọng lực chi tiết trên phạm vi lãnh thổ một quốc gia hoặc một khu vực.

Bên cạnh các lợi ích nêu trên, đối với mạng lưới thống nhất đa quốc gia, việc sử dụng các đại lượng địa thế năng còn có vai trò ràng buộc các mạng lưới độ cao của các quốc gia trên một mặt khởi tính (datum surface) thống nhất - đó là mặt Geoid với thế năng trọng 
trường thực $\mathrm{W}_{0}$ (Fortes P., Lauría E., Brunini C., Amaya W., Sánchez L., Drewes H., Seemller W. (2006b)).

Tuy nhiên để bình sai các mạng lưới độ cao hạng I, II quốc gia với các tham số ẩn là các đại lượng địa thế năng của các mốc độ cao trong hệ độ cao dựa trên mặt Geoid, chúng ta phải giải quyết nhiều vấn đề như chuyển chênh cao đo trong trường trọng lực thực của Quả đất về hiệu các đại lượng địa thế năng giữa hai mốc độ cao trong trường trọng lực chuẩn của ellipsoid quy chiếu, xác định thứ nguyên của đại lượng địa thế năng, xác định các hạn sai cho hiệu các đại lượng địa thế năng và xác định trọng số của các hiệu các đại lượng địa thế năng của các đường thủy chuẩn. Những vấn đề nêu trên không được trình bày và phân tích trong các tài liệu nước ngoài và sẽ được xem xét giải quyết trong bài báo khoa học này.

\section{Giải quyết vấn đề}

2.1. Xác định hiệu các đại lượng địa thế năng giữa hai mốc độ cao trong trường trọng lực chuẩn của ellipsoid quy chiếu

Do độ cao chuẩn $H^{\gamma}$ của mốc độ cao được xác định trong trường trọng lực chuẩn của ellipsoid, nên từ (1) chúng ta suy ra công thức xác định hiệu các đại lượng địa thế năng $d C_{i J}$ giữa hai mốc độ cao i và j:

$$
\begin{aligned}
& d C_{i j}=C_{j}-C_{i}=\bar{\gamma}_{j} \cdot H_{j}^{\gamma}-\bar{\gamma}_{i} \cdot H_{i}^{\gamma}=\left(\frac{\bar{\gamma}_{j}+\bar{\gamma}_{i}}{2}\right) \cdot\left(H_{j}^{\gamma}-H_{i}^{\gamma}\right)+\frac{\bar{\gamma}_{j}}{2} \cdot H_{j}^{\gamma}-\frac{\bar{\gamma}_{i}}{2} \cdot H_{i}^{\gamma}+ \\
& +\frac{\bar{\gamma}_{j}}{2} \cdot H_{i}^{\gamma}-\frac{\bar{\gamma}_{i}}{2} \cdot H_{j}^{\gamma}=\bar{\gamma}_{i j} \cdot h_{i j}^{\gamma}+\bar{H}_{i j}^{\gamma} \cdot\left(\bar{\gamma}_{j}-\bar{\gamma}_{i}\right),
\end{aligned}
$$

ở đây:

$\bar{\gamma}_{i j}=\frac{\bar{\gamma}_{j}+\bar{\gamma}_{i}}{2}-$ giá trị trung bình của các giá trị $\bar{\gamma}_{i}$ và $\bar{\gamma}_{j}$ giữa hai mốc độ cao i và j;

$h_{i j}^{\gamma}=H_{j}^{\gamma}-H_{i}^{\gamma}$ - chênh cao giữa hai mốc độ cao i và j trong trường trọng lực chuẩn của ellipsoid quy chiếu;

$\bar{H}_{i j}^{\gamma}=\frac{H_{j}^{\gamma}+H_{i}^{\gamma}}{2}$ - độ cao chuẩn trung bình giữa hai mốc độ cao i và j.

Trong thực tế tính toán, độ cao chuẩn trung bình $\bar{H}_{i j}^{\gamma}$ giữa hai mốc độ cao i và j được xác định từ các giá trị khái lược của các độ cao chuan của các mốc độ cao này. Trong trường hợp giá trị trung bình $\bar{\gamma}$ của gia tốc lực trọng trường chuẩn của mốc độ cao được biểu diễn trong đơn vị k.Gal, giá trị này được xác định theo công thức:

$$
\bar{\gamma}=\gamma_{0}-0.1543 \cdot 10^{-6} \cdot H^{\gamma}+0.036 \cdot 10^{-12}\left(H^{\gamma}\right)^{2}<k G a l>,
$$

Ở đây gia tốc lực trọng trường chuẩn $\gamma_{0}$ của mặt ellipsoid quy chiếu WGS84 trong đơn vị kGal được xác định theo công thức: 


$$
\gamma_{0}=0.97803253359 \cdot\left(1+0.0053023132 \cdot \sin ^{2} B-0.00000581794875 \cdot \sin ^{2} 2 B\right)<k G a l>\text {, }
$$

độ cao chuẩn $H^{\gamma}$ của mốc độ cao có đơn vị $\mathrm{m}$, còn $\mathrm{B}$ - vĩ độ trắc địa của mốc độ cao.

Trong trường hợp các giá trị trung bình của gia tốc lực trọng trường chuẩn của các mốc độ cao i và j có đơn vị $\mathrm{kGal}$, chênh cao $h_{i j}^{\gamma}$ và độ cao chuẩn trung bình $\bar{H}_{i j}^{\gamma}$ giữa hai mốc độ cao $\mathrm{i}$ và $\mathrm{j}$ có đơn vị $\mathrm{m}$, thì hiệu các đạı ıượng địa thế năng $d C_{i j}$ giữa haı mốc độ cao $\mathrm{i}$ và j trong công thức (2) có đơn vị là kGal.m.

Chênh cao $h_{i j}^{\gamma}$ giữa hai mốc độ cao $\mathrm{i}$ và $\mathrm{j}$ trong trường trọng lực chuẩn của ellipsoid quy chiếu trong công thức (2) được xác định từ việc chuyển chênh cao đo $h_{i j}$ giữa hai mốc độ cao này từ trường trọng lực thực của Quả đất về trường trọng lực chuẩn của ellipsoid quy chiếu theo công thức (Pellinen L.P. (1978); Quy phạm xây dựng lưới độ cao Nhà nước hạng 1,2,3 và 4(1988)):

$$
h_{i j}^{\gamma}=h_{i j}-\frac{\bar{H}_{i j}^{\gamma}}{\bar{\gamma}_{i j}} \cdot\left(\Delta \gamma_{0}\right)_{i j}+\frac{(g-\gamma)_{i j} \cdot h_{i j}}{\bar{\gamma}_{i j}},
$$

ở đây chênh cao đo $h_{i j}$ đã được hiệu chỉnh bởi số cải chính do biến dạng nhiệt của chiều dài trung bình 1 mét của cặp mia; $\left(\Delta \gamma_{0}\right)_{i j}=\left(\gamma_{0}\right)_{j}-\left(\gamma_{0}\right)_{i}$, còn các giá trị $\left(\gamma_{0}\right)_{j}, \quad\left(\gamma_{0}\right)_{i}$ của các mốc độ cao j và i được xác định theo công thức (3); $(g-\gamma)_{i j}$ - giá trị trung bình của dị thường trọng lực chân không giữa hai mốc độ cao i và j aược xac định trong đơn vị kGal.

Trong công thức (4): thành phần $-\frac{\bar{H}_{i j}^{\gamma}}{\bar{\gamma}_{i j}} \cdot\left(\Delta \gamma_{0}\right)_{i j}$ là số cải chính do sự không song song của các mặt đẳng thế trọng trường chuẩn, còn thành phần $\frac{(g-\gamma)_{i j} \cdot h_{i j}}{\bar{\gamma}_{i j}}$ là số cải chính do độ chênh của trọng trường thực của Quả đất so với trọng trương cnuan của ellipsoid quy chiếu (Zakatov P.X. (1964)).

Từ công thức (3) chúng ta thấy rằng:

$$
\bar{\gamma}_{j}-\bar{\gamma}_{i}=\left(\gamma_{0}\right)_{j}-\left(\gamma_{0}\right)_{i}-0.1543 .10^{-6} \cdot h_{i j} \cdot=\left(\Delta \gamma_{0}\right)_{i j}-0.1543 \cdot 10^{-6} \cdot h_{i j} .
$$

Lưu ý các công thức (4) và (5), chúng ta viết lại công thức (2) dưới dạng sau:

$$
d C_{i j}=\left[\bar{\gamma}_{i j}-0.1543 .10^{-6}+(g-\gamma)_{i j}\right] \cdot h_{i j} .
$$

Đến đây chúng ta đã nhận được công thức chuyển chênh cao đo $h_{i j}$ (đơn vị m) thành hiệu các đại lượng địa thế năng $d C_{i j}$ (đơn vị kGal.m) giữa hai mốc độ cao kề nhau i và j. Trong thực tế ở Việt Nam, chênh cao đo $h_{i j}$ đã được chuyển về chênh cao $h_{i j}^{\gamma}$ trong trọng 
trường chuẩn của ellipsoid quy chiếu theo công thức (4), nên để nhận được hiệu các đại lượng địa thế năng $d C_{i j}$ chúng ta sẽ sử dụng công thức (2).

Tại cuộc họp 1983 IAG/UGG của Hội Trắc địa quốc tế (International Association of Geodesy - IAG) năm 1983 tại Hamburg đã ra Nghị quyết 16 [International Association of Geodesy (IAG). (1984)], theo đó các hệ thống được sử dụng trong Trắc địa phải tương ứng với Hệ triều 0 (Zero - Tide System). Trong khi đó chênh cao đo $h_{i j}$ lại được xác định trong hệ triều trung bình (Mean - Tide System). Như vậy hiệu các đại lượng địa thế năng $d C_{i j}$ giữa hai mốc độ cao kề nhau i và $\mathrm{j}$ trong công thức (6) cần phải được chuyển từ hệ triều trung bình về hệ triều 0 theo công thức:

$$
d C_{i j}=\left[\bar{\gamma}_{i j}-0.1543 .10^{-6}+(g-\gamma)_{i j}\right] \cdot h_{i j}+\delta d C_{i j},
$$

ở đây số cải chính $\delta d C_{i j}$ để chuyển hiệu các đại lượng địa thế năng $d C_{i j}$ từ hệ triều trung bình về hệ triều 0 được xác định theo công thức (Mkinen J.(2008)):

$$
\delta d C_{i j}=-0.28841 .\left(\sin ^{2} B_{j}-\sin ^{2} B_{i}\right)-0.00195 .\left(\sin ^{4} B_{j}-\sin ^{4} B_{i}\right) \quad<\text { kGal.m>, }
$$
còn $B_{i}$ và $B_{j}$ là các vĩ độ trắc địa của các mốc độ cao i và j.

\subsection{Lựa chọn đơn vị cho đại lượng địa thế năng}

Do giá trị trung bình của gia tốc lực trọng trường chuẩn $\bar{\gamma}$ của một mốc độ cao có giá trị lớn khi biểu diễn trong đơn vị mGal, nên hiệu các đại lượng địa thế năng $d C_{i j}$ trong công thức (7) với đơn vị mGal.m sẽ có giá trị lớn và điều này dẫn đến sự không tiện lợi khi bình sai mạng lưới độ cao quốc gia trong hệ độ cao dựa trên mặt Geoid với các trị đo là các hiệu các đại lượng địa thế năng giữa các mốc độ cao. Do đó các nhà trắc địa Châu Âu đã thống nhất biểu diễn hiệu các đại lượng địa thế năng $d C_{i j}$ trong đơn vị kGal.m. Điều này có nghĩa là giá trị trung bình của gia tốc lực trọng trường chuẩn được xác định trong công thức (3) phải có đơn vị $\mathrm{kGal}$. Trong trường hợp này, do $1 \mathrm{kGal}=10 \mathrm{~m} \cdot \mathrm{s}^{-2}$, nên thế năng trọng trường thực của mặt Geoid cục bộ tại trạm nghiệm triều Hòn Dấu có giá trị như sau:

$$
\mathrm{W}_{0}=62636847.2911 \mathrm{~m}^{2} . \mathrm{s}^{-2}=6263684.72911 \mathrm{kGal} . \mathrm{m} \text {. }
$$

Theo tài liệu (Hà Minh Hòa và nnk (2012e)) giá trị trung bình của gia tốc lực trọng trường chuẩn trên lãnh thổ Việt Nam bằng $\bar{\gamma}_{V N}=0.9784358 \mathrm{kGal}$. Chúng ta sử dụng giá trị này nhân với các hạn sai trong đơn vị $\mathrm{mm} / \mathrm{km}$ hoặc $\mathrm{mm}$ được cho trong các quy phạm thủy chuẩn (Quy phạm Thủy chuẩn hạng I, II, III và IV (1974); Quy phạm xây dựng lưới độ cao Nhà nước hạng 1,2,3 và 4 (1988)). Trong trường hợp này các hạn sai được quy định trong các Quy phạm đều không thay đổi, chỉ có thay đơn vị $\mathrm{mm} / \mathrm{km}$ bằng $\mathrm{kGal} . \mathrm{mm} / \mathrm{km}$, thay đơn vị mm bằng kGal.mm. Ví dụ, đối với các hạn sai của các sai số ngẫu nhiên, sai số hệ thống và sai số khép trong các đa giác khép kín (Quy phạm Thủy chuẩn hạng I, II, III và IV (1974) được trình bày ở bảng 1.

Sai số trung phương của chênh cao đo (đơn vị $\mathrm{mm}$ ) của đường thủy chuẩn hạng I được xác định theo công thức:

$$
m_{I}=\sqrt{\left(\eta_{I}^{2}+\sigma_{I}^{2} \cdot\left(L_{m}\right)_{I}\right) L_{I}}
$$


Bảng 1

\begin{tabular}{|c|c|c|c|}
\hline \multirow[b]{2}{*}{ Cấp hạng } & \multicolumn{2}{|c|}{ Sai số trung phương giới hạn } & \multirow{2}{*}{$\begin{array}{l}\text { Sai số khép cho phép } \\
\text { trong các đa giác khép } \\
\text { kín }\end{array}$} \\
\hline & $\begin{array}{c}\text { Ngẫu nhiên } \\
\eta(\mathrm{kGal} . \mathrm{mm} / \mathrm{km})\end{array}$ & $\begin{array}{c}\text { Hệ thống } \\
\sigma(\mathrm{kGal} . \mathrm{mm} / \mathrm{km})\end{array}$ & \\
\hline I & 0,8 & 0,08 & $3 \mathrm{kGalmm} \cdot \sqrt{L_{K n}}$ \\
\hline II & 2,0 & 0,20 & $5 \mathrm{kGalmm} \cdot \sqrt{L_{X m}}$ \\
\hline
\end{tabular}

ở đây $\eta_{I}$ - sai số ngẫu nhiên trên $1 \mathrm{~km}$ đường thủy chuẩn hạng $\mathrm{I} ; \sigma_{I}$ - sai số hệ thống trên $1 \mathrm{~km}$ dường thủy chuẩn hạng $\mathrm{I} ;\left(L_{m}\right)_{\mathrm{I}}$ - chiều dài trung bình của tât cả các đường thủy chuẩn hạng I trong mạng lưới độ cao Nhà nước' $\mathrm{L}_{\mathrm{I}}$ - chiều dài của đường thủy chuẩn hạng I với đơn vị km.

Tương tự, sai số trung phương của chênh cao đo (đơn vị $\mathrm{mm}$ ) của đường thủy chuẩn hạng II được xác định theo công thức:

$$
m_{I I}=\sqrt{\left(\eta_{I I}^{2}+\sigma_{I I}^{2} \cdot\left(L_{m}\right)_{I I}\right) \cdot L_{I I}},
$$

ở đây $\eta_{I I}$ - sai số ngẫu nhiên trên $1 \mathrm{~km}$ đường thủy chuẩn hạng II; $\sigma_{I I}$ - sai số hệ thống trên $1 \mathrm{~km}$ đường thủy chuẩn hạng II; $\left(L_{m}\right)_{\mathrm{II}}$ - chiều dài trung bình của tât cả các đường thủy chuẩn hạng II trong mạng lưới độ cao Nhà nước; $\mathrm{L}_{\mathrm{II}}$ - chiều dài của đường thủy chuẩn hạng II với đơn vị km.

Trong trường hợp bình sai hỗn hợp các đường thủy chuẩn hạng I, II, để xác định trọng số của các đường thủy chuẩn hạng I, II chúng ta chọn sai số trung phương của chênh cao trên 1 km đường thủy chuẩn hạng $\mathrm{I}$.

$$
\mu_{I}=\sqrt{\eta_{I}^{2}+\sigma_{I}^{2} \cdot\left(L_{m}\right)_{I}}
$$

làm sai số trung phương đơn vị trọng số.

Đơn vị của đại lượng $\mu_{I}$ là $\mathrm{mm} / \mathrm{km}$. Khi đó trọng số của chênh cao đo của đường thủy chuẩn hạng I được xác định theo công thức:

$$
P_{I}=\frac{\mu_{I}^{2}}{L_{I}},
$$

còn đối với đường thủy chuẩn hạng II trọng số của chênh cao đo được xác định theo công thức:

$$
P_{I I}=\frac{\mu_{I}^{2}}{\left(\eta_{I I}^{2}+\bar{\sigma}_{I I}^{2} \cdot\left(L_{m}\right)_{I I}\right) L_{I I}},
$$


Bây giờ, nếu chúng ta nhận giá trị trung bình của gia tốc lực trọng trường chuẩn ở Việt Nam $\bar{\gamma}_{V N}=0.9784358 \mathrm{kGal}$ với đại lượng $\mu_{I}$, chúng ta sẽ nhận được sai số trung phương hiệu các đại lượng địa thế năng trên $1 \mathrm{~km}$ đường thủy chuẩn hạng $\mathrm{I}$ $\left(\mu_{I}\right)_{d C}=0.9784358 . \mu_{I}$ với đơn vị là $\mathrm{kGal} . \mathrm{mm} / 1 \mathrm{~km}$. Tương tự, nhân giá trị $\bar{\gamma}_{V N}$ với sai số trung phương $\mathrm{m}_{\mathrm{I}}$ (đơn vị mm) của chênh cao đường hạng I và sai số trung phương $\mathrm{m}_{\mathrm{II}}$ (đơn vị mm) của chênh cao đường hạng II, chúng ta sẽ nhận được sai số trung phương $\left(\mathrm{m}_{\mathrm{I}}\right)_{\mathrm{dC}}$ của hiệu các đại lượng địa thế năng của đường thủy chuẩn hạng I và sai số trung phương $\left(\mathrm{m}_{\mathrm{II}}\right)_{\mathrm{dC}}$ của hiệu các đại lượng địa thế năng của đường thủy chuẩn hạng II, thêm vào đó các sai số trung phương này có đơn vị kGal.mm. Không khó khăn để nhận thấy rằng trọng số của hiệu các đại lượng địa thế năng của đường hạng I có giá trị tương tự như trong công thức (7) và trọng số của hiệu các đại lượng địa thế năng của đường hạng II có giá trị tương tự như trong công thức (8).

Như vậy, khi bình sai hỗn hợp các mạng lưới hạng I, II quốc gia trong hệ độ cao dựa trên mặt Geoid với các trị đo là các hiệu của các đại lượng địa thế năng của các đường thủy chuẩn hạng I, II, các trọng số của các hiệu của các đại lượng địa thế năng tương tự như trong trường hợp bình sai với các chênh cao đo của các đường thủy chuẩn hạng I, II. Tuy nhiên, sai số trung phương đơn vị trọng số sau bình sai là sai số trung phương của hiệu của các đại lượng địa thế năng trên $1 \mathrm{~km}$ đường thủy chuẩn và có đơn vị $\mathrm{kGal} . \mathrm{mm} / 1 \mathrm{~km}$.

Các tham số ẩn tìm được là các giá trị bình sai của các đại lượng địa thế năng của các mốc độ cao. Độ cao chuẩn sau bình sai của mốc độ cao $\mathrm{M}$ bất kỳ được xác định theo công thức (1).

Khi đã biết giá trị bình sai của đại lượng địa thế năng $\mathrm{C}_{\mathrm{M}}$ của mốc độ cao $\mathrm{M}$, chúng ta sẽ xác định được thế năng trọng trường thực của mốc độ cao $M$ theo công thức:

$$
W_{M}=W_{0}-C_{M}
$$

\section{Kết luận}

Bài báo khoa học này đã phân tích và xây dựng được công thức chuyển chênh cao đo từ trường trọng lực thực của Quả đất thành hiệu các đại lượng địa thế năng giữa hai mốc độ cao trong trường trọng lực chuẩn của ellipsoid quy chiếu. Điều này cho phép sử dụng các hiệu các đại lượng địa thế năng giữa các mốc độ cao như các trị đo để bình sai mạng Iưới độ cao hạng I, II quốc gia trong hệ độ cao dựa trên mặt Geoid. Các kết quả nghiên cứu trong bài báo cũng chỉ ra rằng khi biểu diễn giá trị trung bình của gia tốc lực trọng trường chuẩn của mốc độ cao trong đơn vị $\mathrm{k} G a l$, hiệu các đại lượng thế năng và thế năng trọng trường thực trong đơn vị kGal.m, các hạn sai quy định trong quy phạm không thay đổi giá trị, chỉ có thứ nguyên thay đổi từ $\mathrm{mm} / \mathrm{km}$ thành $\mathrm{kGal} . \mathrm{mm} / \mathrm{km}$, từ $\mathrm{mm}$ thành $\mathrm{kGal} . \mathrm{mm}$. Cũng tương tự, trọng số của hiệu các đại lượng địa thế năng của đường độ cao về giá trị bằng trọng số của chênh cao của đường độ cao đó. Như vậy, việc thay chênh cao đo bằng hiệu các đại lượng địa thế năng giữa hai mốc độ cao không làm thay đổi đáng kể các quy định kỹ thuật hiện hành và quy trình bình sai mạng lưới độ cao hạng I, II quốc gia.O 


\section{Tài liệu tham khảo}

[1]. Zakatov P.X. (1964). Trắc địa cao cấp. Trắc địa cầu, Trắc địa lý thuyết và các cơ sở của trọng lực. Matxcơva, Nedra, 504 trg. (Tiếng Nga).

[2]. Pellinen L.P. (1978). Trắc địa cao cấp (trắc địa lý thuyết). Matxcơva, Nedra, 264 trg. (Tiếng Nga).

[3]. Quy phạm Thủy chuẩn hạng I, II, III và IV (1974). Tổng cục Trắc địa và Bản đồ trực thuộc Hội đồng Bộ trưởng Liên Xô. Matxcơva, Nedra, 1974, 160 trg. (Tiếng Nga).

[4]. International Association of Geodesy (IAG). (1984). IAG Resolutions adopted at the XVIII General Assembly of the IUGG in Hamburg, August 1983. Bulletin Geodetique, 58(3), "The Geodesist's handbook", p. 321.

[5]. Quy phạm xây dựng lưới độ cao Nhà nước hạng 1,2,3 và 4 (1988). Cục Đo đạc và Bản đồ Nhà nước. Hà Nội - 1988.

[6]. Bursa M., Kenyon S., Kouba J., Muller A., Radej K., Vatrt V., Vojtiskova M., Vitek V. (1999). Long - Term Stability of Geoidal Geopotential from TOPEX./POSEIDON Satellite Altimetry 1993 - 1999. Earth, Moon and Planets, 84, 3, pp. 163-176.

[7]. Bursa M., Kenyon S., Kouba J., Radëi K., Vatrt V., Vojtiskova M., Simek J. (2002). Word Height System specified by geopotential at tide gauge stations. IAG Symposia 124, 291-296. Springer.

[8]. Bursa M., Kenyon S., Kouba J., Sima Z., Vatrt V., Vitek V. and Vojtiskova M. (2007). The geopotential value $W_{0}$ for specifying the relativistic atomic time scale and a global vertical reference system. J. of Geodesy, 81, 2, pp. 103-110.

[9]. Dennis D. McCarthy, Gerard Petit. (2004). IERS Conventions (2003). IERS Technical Note No 32. Frankfurt am Main, 2004.

[10]. Sánchez, L. (2005). Definition and Realization of the SIRGAS Vertical Reference System within a Globally Unified Height System. Presented at the IAG Scientific Assembly, Cairns, Australia. August 22-26. IAG Symposia in print.

[11]. Fortes P., Lauría E., Brunini C., Amaya W., Sánchez L., Drewes H., Seemuller W. (2006). SIRGAS - a geodetic enterprise. Scientific work of the field Geodesy and Geoinformatic of the University of Hanover, J. of Coordinates, Vol. 258, pp. 59-70, 2006.

[12]. Sacher M., Ihde J., Liebsch G., Mkinen J. (2008). EVRF2007 as Realization of the European Vertical Reference System. EUREF Symposium, June 17 - 21 2008, Brussels.

[13]. Mä kinen J. (2008). The treatment of permanent tide in EUREF products. Paper presented at the Symposium of the IAG Sub-commission for Europe (EUREF) in Brussels, June $17-21,2008$. Submitted to the proceedings.

[14]. Sideris Michael G., Spiros Pagiatakis. (2010). A Geoid-Based Vertical Reference Frame for Height Modernization in North America. Project number SII-PIV-50, PHASE IV (2009-2012).

[15]. M. Veronneau, J. Huang. (2007). Moving to a Modernized Height Reference System in Canada: Rationale, Status and Plans. Natural Resources Canada. American Geophysical Union, Spring Meeting 2007. 1214 Geopotential theory and determination 
(0903), 1229 Reference systems. 007AGUSM.G33B.05V.

[16]. Petit G., Luzum B. (2010). IERS Conventions (2010). IERS Technical Note No 36, Verlag dés Bundesamts fur Kartographie und Geodasie. Frankfurt am Main 2010, 179 pp.

[17]. Daniel Roman and Neil Weston (2012). Beyond GEOID12: Implementing a New Vertical Datum for North America. FIG Working Week 2012: Knowing to manage the territory, protect the environment, evaluate the cultural heritage, Rome, Italia, 6-10 May 2012.

[18]. Hà Minh Hòa. (2007). Giải quyết một số vấn đề liên quan đến việc chuyển hệ độ cao được xác định từ mặt nước biển trung bình ở trạm thủy triều về mặt Quasigeoid toàn cầu. Tạp chí Địa chính số 2 , tháng 4/2007, trg. 3 - 11 .

[19]. Hà Minh Hòa (2012a). Các phương hướng hoàn thiện hệ độ cao quốc gia. Tạp chí Khoa học Đo đạc và Bản đồ, No11, 03/2012, trg. 1 -9.

[20]. Hà Minh Hòa (2012b). Xây dựng hệ độ cao dựa trên mặt Geoid gắn kết với việc xây dựng Hệ tọa độ động lực quốc gia. Tạp chí Khoa học Đo đạc và Bản đồ, No12, 06/2012, trg. 1 - 12.

[21]. Hà Minh Hòa (2012c). Nghiên cứu lựa chọn Hệ độ cao dựa trên mặt Geoid để giải quyết bài toán hoàn thiện Hệ độ cao gắn liền với việc xây dựng mô hình Quasigeoid độ chính xác cao. Tạp chí Khoa học Đo đạc và Bản đồ, No13, 09/2012, trg. 1 - 8.

[22]. Hà Minh Hòa (2012d). Nghiên cứu xác định thế năng trọng trường thực $W_{0}$ của mặt Geoid cục bộ trùng với mặt biển trung bình tại trạm nghiệm triều Hòn Dấu. Báo cáo khoa học. Tuyển tập báo cáo Hội nghị Khoa học và Công nghệ "Trắc địa và Bản đồ vì sự nghiệp tài nguyên và Môi trường". Viện Khoa học Đo đạc và Bản đồ - Hội Trắc địa, Bản đồ và Viển thám Việt Nam. Hà Nội - Tháng 10/2012. Trg. 6-19.

[23]. Hà Minh Hòa và nnk (2012e). Nghiên cứu cơ sở khoa học của việc hoàn thiện Hệ độ cao gắn liền với việc xây dựng Hệ tọa độ động lực quốc gia. Đề tài khoa học và công nghệ cấp Bộ Tài nguyên và Mồi trường giai đoạn 2010 - 2012. Hà Nội - 2012.O

\section{Summary}

SOLVING A SOME TECHNO - SCIENTIFIC PROBLEMS RELATED TO MATHEMATICAL PROCESSING OF THE STATE HEIGHT NETWORK IN THE GEOID - BASED VERTICAL REFERENCE SYSTEM

\section{Assoc. Prof. Dr. Sc. Ha Minh Hoa, MSc. Nguyen Thi Thanh Huong \\ Vietnam Institute of Geodesy and Cartography}

This scientific article is focused to construction of formula for transformation of the differential leveling observation between two benchmarks to the difference of geopotential numbers to serve an adjustment of state height network in the geoid - based vertical reference system. This article also shows that when mean value of normal gravity acceleration corresponding to benchmark has the unit of $\mathrm{kGal}$, difference of geopotential numbers and Earth potential will have the unit of $\mathrm{kGal} . \mathrm{m}$. On this occasion error limits gave on Instruction of the state height network will not change values, only their units will be changed from $\mathrm{mm}$ to $\mathrm{kGal} . \mathrm{mm}$, from $\mathrm{mm} / 1 \mathrm{~km}$ to $\mathrm{kGal} . \mathrm{mm} / 1 \mathrm{~km}$. Analogically, weight of the difference of geopotential numbers of leveling line will be equal to weight of the differential leveling observation of that leveling line. 0

Ngày nhận bài 04/3/2013. 